\title{
LAPTM4B-35 expression is associated with pathological grades and clinical stages in salivary adenoid cystic carcinoma
}

\author{
JIANLIN FAN ${ }^{1}$, JIANXIN YANG $^{1}$, WEIPING QIAO ${ }^{2}$, WEI LIU ${ }^{3}$ and CHUNGEN XING ${ }^{4}$ \\ ${ }^{1}$ Department of Stomatology, The Second Affiliated Hospital of Soochow University, Suzhou, Jiangsu 215004; \\ ${ }^{2}$ Department of Stomatology, Wuzhong People's Hospital, Suzhou, Jiangsu 215100; ${ }^{3}$ Department of Pathology, \\ The First Affiliated Hospital of Soochow University, Suzhou, Jiangsu 215006; ${ }^{4}$ Department of General Surgery, \\ The Second Affiliated Hospital of Soochow University, Suzhou, Jiangsu 215004, P.R. China
}

Received April 1, 2019; Accepted September 9, 2019

DOI: $10.3892 / \mathrm{ol} .2019 .11124$

\begin{abstract}
Salivary adenoid cystic carcinoma is one of the most common malignancies of the head and neck. The lysosome-associated protein transmembrane- $4 \beta$ gene $(L A P T M 4 B)$ is a novel oncogene that has been found overexpressed in a number of clinically aggressive cancers. This study aimed to investigate the expression of the LAPTM4B-35 protein in normal salivary gland and salivary adenoid cystic carcinoma, a relatively indolent malignancy, and explore its clinicopathological significance in this malignancy. By immunohistochemical analysis, LAPTM4B-35 expression was evaluated in 106 cancer tissues, their adjacent non-cancerous tissues and five normal salivary glands. The correlation of LAPTM4B-35 expression with clinicopathological parameters was assessed using Chi-square or Fisher's exact test. The level of LAPTM4B-35 expression varied among different cell types of normal salivary glands. It was expressed at a fairly low level in serous and mucous acini, at low level in intercalated duct and excretory duct cells and moderately in secretory/striated ducts. In $50 \%$ of high grade tumor tissues tested, LAPTM4B-35 was markedly overexpressed. LAPTM4B-35 levels were significantly associated with histological grade and clinical stage. LAPTM4B-35 plays an important role in salivary adenoid cystic carcinoma and may serve as a diagnostic marker and a target for individualized therapy.
\end{abstract}

Correspondence to: Dr Chungen Xing, Department of General Surgery, The Second Affiliated Hospital of Soochow University, 1055 Sanxiang Road, Suzhou, Jiangsu 215004, P.R. China

E-mail: xigbdm9837@163.com; chungenxingsz@sina.com

Dr Wei Liu, Department of Pathology, The First Affiliated Hospital of Soochow University, 899 Pinghai Road, Suzhou, Jiangsu 215006, P.R. China

E-mail: wliu3sz@sina.com

Key words: LAPTM4B-35, salivary adenoid cystic carcinoma, histological grade, clinical stage

\section{Introduction}

Adenoid cystic carcinoma (ACC) is a relatively uncommon malignant tumor in various gland-bearing organs, most commonly in the salivary glands. It accounts for $\sim 1 \%$ of all malignant head and neck tumors and $10 \%$ of all salivary gland tumors (1). ACC of the salivary gland (SACC) occurs more commonly in the major salivary glands and is reported to be the most common cancer of the minor salivary glands (2). Histologically, SACC is presumed to originate from the intercalated duct. It is composed of epithelial and myoepithelial neoplastic cells that form various patterns, including tubular, cribriform, and solid form. SACC is characterized by a high propensity to invade nerves and an apparently indolent clinical course, with frequent local recurrences, a late onset of metastases and often a poor long-term survival. The genetics of primary SACC have been extensively investigated, which has resulted in the identification of recurrent gene fusions involving the $M Y B$ and $M Y B L 1$ genes (3). However, as most SACCs are resistant to radiation therapy, the identification of additional prognostic biomarkers is needed to predict the response of SACC to targeted therapy and/or identify targets for individual therapy.

Lysosome-associated protein transmembrane $4 \beta$ (LAPTM4B), a newly identified oncogene (NM_018407, Gene ID: 55353), was first cloned in human hepatocellular carcinoma (HCC) (4). It is located on chromosome 8q22.1, spanning at least $50 \mathrm{~kb}$, including seven exons and six introns. The full-length cDNA of LAPTM4B contains two translational initiation codons (ATG) with an interval of 273 base pairs, and encodes at least two protein isoforms, LAPTM4B-35 and LAPTM4B-24, a type III transmembrane protein with four transmembrane regions. LAPTM4B-35 differs from LAPTM4B-24; it contains an extra 91 amino acid residues at the $\mathrm{N}$ terminus, which harbors a proline-rich domain, PPRP. This serves as the binding site of the SH3 domain of some signaling molecules and plays critical roles in the proliferation and metastatic potential of tumor cells (5). The LAPTM $4 B$ gene has been found to be expressed at a fairly low level in normal human tissues except the testis and muscles, but the LAPTM4B-35 protein is upregulated in various types of carcinomas. The overexpression of LAPTM4B-35, rather than 
LAPTM4B-24, has been suggested to be closely associated with high-grade HCC (6), and is inversely correlated with overall survival and disease-free survival of patients with $\operatorname{HCC}(7,8)$, gallbladder carcinoma (9), colorectal carcinoma (10), ovarian carcinoma (11,12), non-small cell lung cancer $(13,14)$, prostate cancer (15), endometrial carcinoma of uterus (16) and gastric cancer $(17,18)$. So far there is no clear evidence suggesting that there are any clinicopathological features associated with upregulation of LAPTM4B-35 in SACC tissues. In the present study, we explored LAPMT4B-35 expression in indolent SACC to identify its potential relationship with clinicopathological features. Our results suggest that LAPTM4B-35 overexpression is associated with high histological grade and advanced clinical stage.

\section{Materials and methods}

General. Archived formalin-fixed, paraffin-embedded samples were obtained from patients with SACC who were surgically treated in The Second Affiliated Hospital of Soochow University and outside institutes between January 2010 and December 2017. The slides were reviewed by two pathologists. The SACC tumors were histopathologically classified as grade I, II or III according to WHO classification; grade I tumors mainly showed only a tubular and cribriform pattern without a solid component; grade II tumors were defined as cribriform with solid components of $<30 \%$; grade III tumors were those showing solid components of $\geq 30 \%$. When there was an area of histological transformation, it was designated as transformed. Any differences in the scores were resolved by discussion between the two pathologists. The Ethics Committee of the Second Affiliated Hospital of Soochow University approved the study. All the patients consented in writing to participate in the study.

Immunohistochemical staining. LAPTM4B-35 expression was detected using immunohistochemistry for paraffin-embedded specimens obtained from 106 patients with SACC. A total of five normal salivary glands and 106 SACC tissues was sectioned at $4 \mu \mathrm{m}$ and stained with $\mathrm{H} \& \mathrm{E}$ for confirmation. Sections adjacent to the H\&E-stained sections were used for LAPTM4B-35 immunohistochemical (IHC) staining. Anti-human LAPTM4B-35 rabbit polyclonal antibody (LAPTM4B-N1-99-pAb), purified by immuno-affinity and specifically recognizing LAPTM4B-35 (but not LAPTM4B-24), was provided by Professor Rou-Li Zhou from the Department of Cell Biology at Peking University Health Science Centre. The IHC analysis was performed as described previously (8). Briefly, the sections were deparaffinized in xylene, rehydrated in ethanol and incubated with $0.3 \%$ hydrogen peroxide $\left(\mathrm{H}_{2} \mathrm{O}_{2}\right)$ for $10 \mathrm{~min}$ to block endogenous peroxidase activity, then non-specific immunoglobulin binding was blocked by incubation with $10 \%$ non-immunized normal rabbit serum for $10 \mathrm{~min}$. After washing in Tris buffer, the slides were incubated for $1 \mathrm{~h}$ at room temperature with the primary rabbit polyclonal anti-LAPTM4B-35 antibody $(1 \mathrm{mg} / \mathrm{ml}$, dilution 1:100). The slides were then washed and incubated for 30 min with biotin-labeled secondary antibody (animal origin: goat, catalog no.: SP-9001). Color development was performed by incubation with horseradish peroxidase-conjugated streptavidin for $45 \mathrm{~min}$, followed by 3,3'-diaminobenzidine tetrahydrochloride (Dako) in $0.01 \% \mathrm{H}_{2} \mathrm{O}_{2}$ for $10 \mathrm{~min}$. Finally, the slides were counterstained with Meyer's hematoxylin for $30 \mathrm{sec}$. IHC was performed using an IHC kit purchased from Jingmei Inc. according to the manufacturer's instructions. Negative control slides were stained with normal rabbit IgG at the same dilution. The positive controls were HCC specimens with a positive expression of LAPTM4B-35.

Semi-quantitative analysis of LAPTM4B-35 staining. IHC analysis of LAPTM4B-35 was performed as previously described (10). All of the stained tissues were reviewed separately by two pathologists experienced in evaluating IHC (W.L. and Q.Y.) who were blinded to the clinical status. LAPTM4B-35 expression was determined semi-quantitatively by the sum of cytoplasm staining intensity and the percentage of positively staining tumor cells. The percentage of immune-reactive tumor cells was scored as follows: 0 represents $<10 \%$; 1 represents $10-50 \% ; 2$ represents $>50 \%$. The staining intensity was scored as 0 , no staining or weak staining; 1 , moderate staining; and 2 , strong staining. The overall score for LAPTM4B-35 expression was the sum of points determined for the percentage of positive cells and staining intensity, and an overall score ranging from 0 to 4 was assigned. For the statistical analysis, the patients were divided into two groups. Scores of 0-2 and 3-4 were, respectively, considered to be low and high/over expression. Any difference in the scoring was resolved by co-observation and discussion between the two pathologists.

Statistical analysis. Statistical analyses were performed using SPSS 16.0 software (SPSS, Inc.). The Chi-square test or the Fisher's exact were used to examine the association between LAPTM4B-35 expression and the clinicopathological factors of the patients. A two-sided $\mathrm{P}<0.05$ was considered statistically significant.

\section{Results}

Demographic characteristics of patients with SACC. A total of 106 cases with SACC were assessed for LAPTM4B-35 expression. There were 45 males and 61 females aged 20-87 years, with a median age of 50 years. The patients were divided into two groups based on their age ( $\leq 50$ and $>50$ years). Of the 106 patients, $43(40.57 \%)$ had tumors categorized as histological grade I, 33 (31.13\%) had grade II tumors, 26 (24.53\%) had grade III tumors, and 4 (3.77\%) cases showed transformation. A standardized neck dissection involving levels I, I to III, or I to IV/V was performed in 22 patients $(20.75 \%)$. There were no surgical resection specimens in 11 cases diagnosed by biopsy only. Thus, some clinical related information, such as tumor size and TNM stage, was not available for these 11 cases. The main characteristics of the patients are presented in Table I.

Expression of LAPTM4B-35 in normal salivary glands. Salivary glands are divided into major and minor glands. According to the type of epithelial cell and their secretory product, the acini are classified in three forms: serous, mucoserous, and mucous. First, we detected the expression of LAPTM4B-35 in five normal salivary glands by means of IHC analysis. Representative examples of LAPTM4B-35 
Table I. Relationship between LAPTM4B-35 overexpression and clinicopathological features of SACC.

\begin{tabular}{|c|c|c|c|c|}
\hline Variables & $\begin{array}{l}\text { Patients } \\
\text { total } \\
\text { (n) }\end{array}$ & $\mathrm{n}$ & $\begin{array}{c}\text { LAPTM4B-35 } \\
\text { overexpression } \\
(\%)\end{array}$ & P-value \\
\hline All cases & 106 & & & \\
\hline \multicolumn{5}{|l|}{ Sex } \\
\hline Male & 45 & 10 & 22.22 & \multirow[t]{2}{*}{0.51} \\
\hline Female & 61 & 17 & 27.87 & \\
\hline \multicolumn{5}{|l|}{ Age (years) } \\
\hline$\leq 50$ & 55 & 17 & 30.91 & \multirow[t]{2}{*}{0.18} \\
\hline$>50$ & 51 & 10 & 19.61 & \\
\hline \multicolumn{5}{|l|}{ Tumor location } \\
\hline Major salivary gland & 47 & 13 & 27.66 & \multirow[t]{2}{*}{0.64} \\
\hline Minor salivary gland & 59 & 14 & 23.73 & \\
\hline \multicolumn{5}{|l|}{ Tumor size } \\
\hline$\leq 2 \mathrm{~cm}$ & 32 & 9 & 28.13 & \multirow[t]{2}{*}{$0.53^{\mathrm{a}}$} \\
\hline$>2 \mathrm{~cm}$ & 63 & 14 & 22.22 & \\
\hline \multicolumn{5}{|l|}{ Histopathological grade } \\
\hline G1 & 43 & 6 & 13.95 & \multirow[t]{3}{*}{0.001} \\
\hline $\mathrm{G} 2$ & 33 & 6 & 18.18 & \\
\hline G3 and transformed & 30 & 15 & 50.00 & \\
\hline \multicolumn{5}{|l|}{ TNM stage } \\
\hline I, II & 77 & 15 & 19.48 & \multirow[t]{2}{*}{$0.026^{\mathrm{a}}$} \\
\hline III, IV & 18 & 8 & 44.44 & \\
\hline \multicolumn{5}{|l|}{ Lymph node metastasis } \\
\hline Absent & 89 & 20 & 22.47 & \multirow[t]{2}{*}{$0.62^{\mathrm{a}}$} \\
\hline Present & 6 & 2 & 33.33 & \\
\hline
\end{tabular}

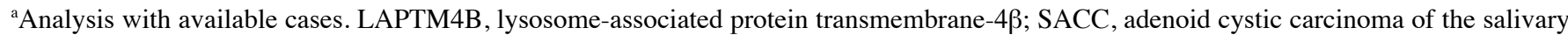
gland.

staining are shown in Fig. 1. In all salivary glands tested, LAPTM4B-35 expression was variable among different parts of the glands and cell types. It was expressed at a very low level in acini, both in serous and mucous acini, at a low level in intercalated duct and excretory duct cells and moderately in secretory/striated ducts (Fig. 1A-F). Consistent with previous studies, the positive LAPTM4B-35 signal was mainly localized in the cell cytoplasm.

LAPTM4B-35 protein expression in SACC and its relationship with clinicopathological characteristics. LAPTM4B-35 expression was low in 79 of 106 patients (74.53\%) and high in 27 of 106 patients $(25.47 \%)$, according to the criteria mentioned above. Importantly, high LAPTM4B-35 expression was identified in 6/43 (13.95\%) of histological grade I samples, 6/33 (18.18\%) of grade II, and 15/30 (50.00\%) of grade III and transformed tumor samples. As shown in Fig. 2, the LAPTM4B-35 immunoreactivity was significantly associated with histological grade ( $\mathrm{P}=0.001$, Fig. 2). We then grouped grade I and II as low grade, and grade III and transformed as high grade. High LAPTM4B-35 expression was higher in high grade SACC (15/30, 50.0\%), compared to $12 / 76(15.8 \%)$ in low grade SACC $(\mathrm{P}=0.0003)$. In addition, as shown in Table I and Fig. 2, a significant difference was also found between high LAPTM4B-35 expression and advanced clinical stage ( $8 / 18$ vs. $15 / 77, \mathrm{P}=0.026)$. As shown in Table I, although no statistically significant differences were identified between LAPTM4B-35 expression and age or lymph node metastasis, there was a trend that a high expression of LAPTM4B-35 was more common in younger patients $(17 / 55,30.91 \%$ vs. $10 / 51,19.61 \%, \mathrm{P}=0.18)$, and lymph node metastasis (2/6, 33.33\% vs. 20/89, 22.47\%, $\mathrm{P}=0.62)$ (Fig. 2). However, no significant association was observed between LAPTM4B-35 immunoreactivity and sex, tumor size or location $(\mathrm{P}>0.05)$.

\section{Discussion}

A number of previous studies have shown that LAPTM4B-35 overexpression occurs with high frequency (62-88\%) in a large number of carcinomas that grow rapidly, and plays critical roles in tumorigenesis, progression, metastasis, recurrence, and drug resistance (5,9-11,13-18). To the best of our knowledge, this is the first report of LAPTM4B-35 expression in a 

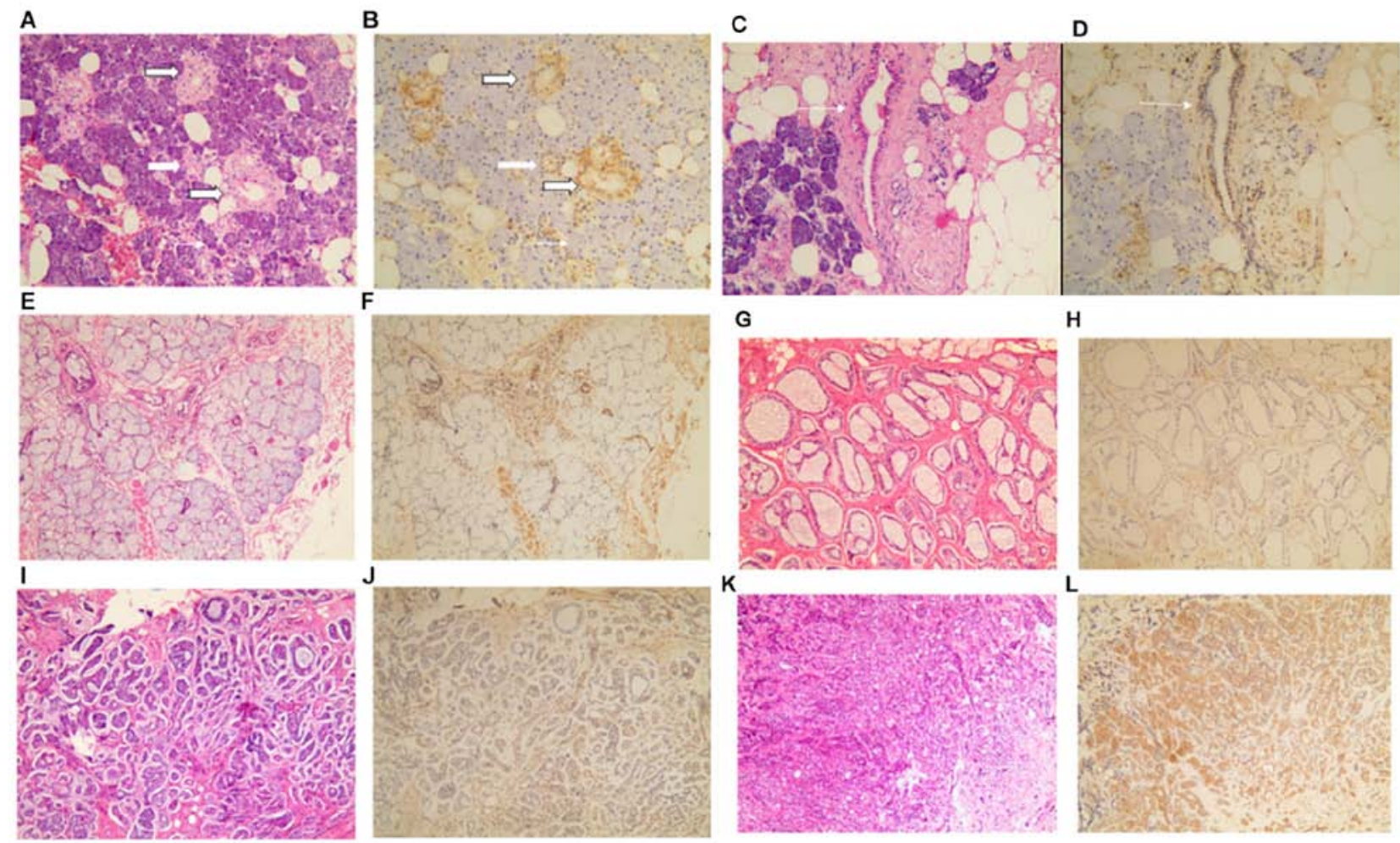

Figure 1. Representative immunohistochemical staining patterns showing the levels of lysosome-associated protein transmembrane-4 $\beta$-35 (LAPTM4B-35) in $4 \mu \mathrm{m}$ thick paraffin-embedded sections of normal and tumour tissue samples from patients with salivary adenoid cystic carcinoma. (A and B) $\rightarrow$ indicates serous acini, $\Rightarrow$ indicates intercalated ducts, $\Rightarrow$ indicates secretory/striated ducts. $(\mathrm{C}$ and $\mathrm{D}) \rightarrow$ indicates excretory duct. $(\mathrm{E}$ and $\mathrm{F}) \rightarrow$ indicates mucous acini. Low expression of LAPTM4B in salivary adenoid cystic carcinoma (SACC, 1H, 1G for H\&E), moderate expression of LAPTM4B-35 (1J, 1I for H\&E) and high expression of LAPTM4B-35 (1L, IK for H\&E). Original magnification, $\mathrm{x} 100$.

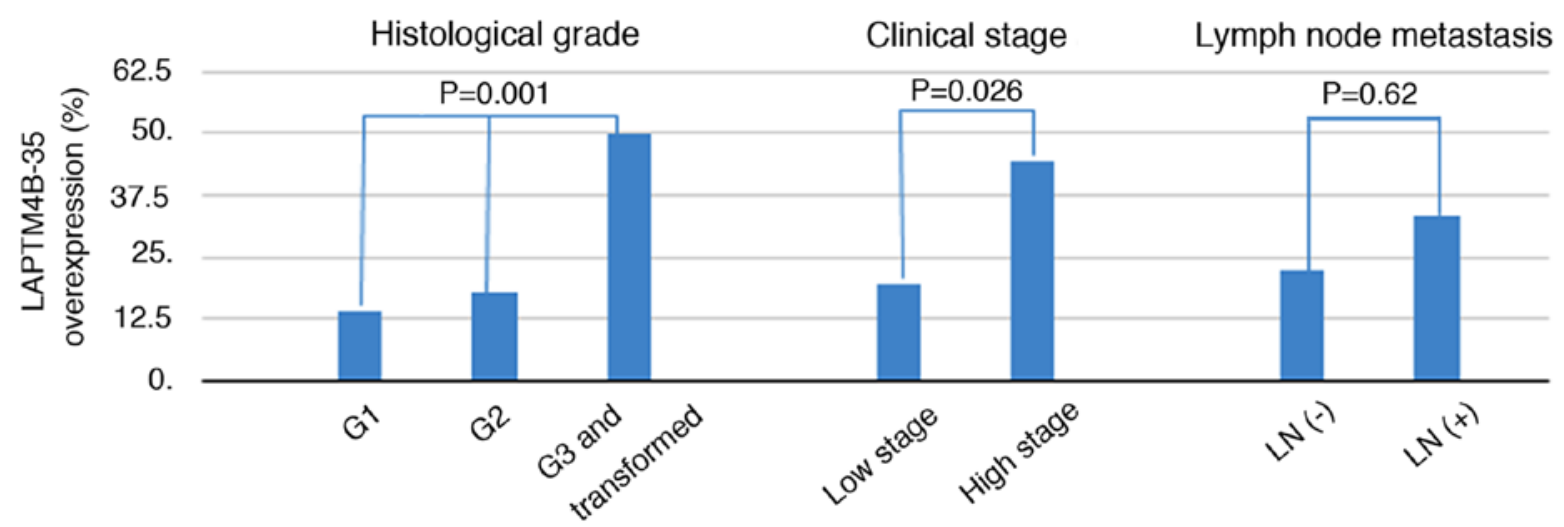

Figure 2. Association of clinicopathological features with LAPTM4B-35 expression. LAPTM4B-35 immunoreactivity was significantly associated with histological grade $(\mathrm{P}=0.001)$ and advanced clinical stage $(\mathrm{P}=0.026)$. Although no statistically significant difference was identified between LAPTM4B-35 expression and lymph node metastasis $(\mathrm{P}=0.62)$, there was a trend that high expression of LAPTM4B-35 was more common in cases with lymph node metastasis. All statistical analyses were performed by Chi-square test or Fisher's exact test.

clinically indolent malignancy, SACC. We found that the total high expression frequency of LAPTM4B-35 in SACC tested was only $25.47 \%$ (27/106). Most SACC express LAPTM4B-35 at relatively low level, but at a high level in $50 \%$ of SACC with high pathological grade and advanced clinical stage. Although growing slowly, SACC is a life-threatening malignancy for its propensity of recurrence and tumor-related death continuously occurring within 30 years after primary treatment. Thus, the identification of novel prognostic biomarkers for SACC is warranted to predict and improve the prognosis of SACC. In this study, we demonstrated an association between high
LAPTM4B-35 expression with clinicopathological parameters of SACC. Previous studies have shown that LAPTM4B-35 expression was dramatically elevated in high grade (above grade III) carcinomas (5,8-11,13-18). In line with previous findings, comparing SACC with the paired compartment (Fig. 1), a higher LAPTM4B-35 protein expression was correlated with more aggressive features, including high histopathological grade and advanced clinical stage. In addition, there was a propensity that a high LAPTM4B-35 expression was associated with younger age of onset or lymph node metastasis. Lymph node metastasis may be underestimated based on the 
uncompleted specimen from lymph nodes and the limited number of cases, which may affect the power of the statistical analyses. A larger sample size remains to be studied to further verify these results (19). There was no association between LAPTM4B-35 expression and sex, age, tumor location or size.

$L A P T M 4 B$ has been reported widely expressed in human tissues with a relatively high expression level in heart, skeletal muscle, and testis, a moderate expression level in ovary, kidney, and pancreas, a low expression level in liver, spleen, and thymus, and the lowest expression in lung and peripheral leukocytes (4). In this study, LAPTM4B-35 showed variable expression level in normal salivary glands. It was negative in acini cells, weak in intercalated duct and excretory duct cells, and moderate in striated ducts cells. The function of LAPTM4B-35 in normal salivary glands remains to be clarified. On the other hand, it is interesting to note that previous studies focused on tumors derived from one cell type. As SACC is composed to two types of cells, the epithelial and myoepithelial neoplastic cells, LAPTM4B-35 might also play an important role in cancers that originate from two cell types. The role of LAPTM4B-35 in epithelial and myoepithelial neoplastic cells merits further investigation.

Conventional prognostic factors for salivary gland carcinomas include advanced stage, close or involved surgical margins, vascular invasion, and high histological grade. However, the infiltrative nature of SACC makes it a complex task to achieve both tumor-free margins and preserve vital structures, function, and acceptable cosmetic outcome. Recently, some significant prognostic factors of SACC, including clinicopathological characteristics and molecular markers, were identified. Interestingly, intraneural but not perineural invasion has been identified to be an independent predictor of poor prognosis (20). In contrast to more common malignancies, only very few molecular-based prognostic studies have been carried out in SACC. Overall, SACC exhibit a low mutational burden. Deletion of the chromosomal region $1 \mathrm{p} 36$ has been identified predominantly in solid type ACC and was associated with an aggressive clinical course (21). NOTCH1 mutations were identified in a subgroup of patients with solid subtype, advanced-stage disease and poor prognosis. Preliminary data showed that a Notch1 inhibitor had antitumor activity and may serve as a potential therapeutic target (22). On the other hand, it has been reported that LAPTM4B-35 is a cancer driver gene, and its high expression plays fundamental oncogenic and onco-progressive roles. It has been shown to be an independent prognostic factor that may be a potential biomarker for prognostic prediction and may be a novel therapeutic target (23). The present study suggests that this may also be true in the context of SACC (24).

There are also some limitations to the present study. First, due to unavailable commercial SACC cell lines, we were not able to perform functional study to support our current findings. Second, formalin fixed paraffin embedded (FFPE) tissue was the only research material in the study. The results could not be validated by western blot analysis or qPCR analysis due to the poor quality of protein and mRNA extracted from FFPE tissue, although previous studies showed increased expression of LPTM4B-35 by both IHC and western blot analysis in ovarian carcinoma (12). Lastly, as SACC is an indolent malignancy and progresses slowly, there is limited follow-up data that could be used for survival analysis. A future study is warranted to evaluate the association between LAPTM4B-35 expression and prognosis using long-term follow-up data.

Taken together, the present study shows that high LPTM4B-35 expression is associated with high histological grade and advanced clinical stage. LAPTM4B-35 may be a subtype-specific marker for SACC and is a potential prognostic biomarker for the management of SACC.

\section{Acknowledgements}

The authors would like to thank Professor Rou Li Zhou for her help in the preparation of the manuscript.

\section{Funding}

This study was supported by grants from the National Natural Science Foundation of China (no. 31100944) and Postdoctoral Science Foundation of Jiangsu Province (no. 1402080B).

\section{Availability of data and materials}

The datasets used and/or analyzed during the present study are available from the corresponding author on reasonable request.

\section{Authors' contributions}

JF wrote the manuscript. JF, JY and WQ led the conception and design of this study. JF, WL and CX were responsible for the data collection and analysis. JY and CX were in charge of interpreting the immunohistochemistry results. JF and WQ helped with statistical analysis. All the authors read and approved the final manuscript.

\section{Ethics approval and consent to participate}

The study was approved by the Ethics Committee of the Second Affiliated Hospital of Soochow University, China. Signed informed consents were obtained from the patients or the guardians.

\section{Patient consent for publication}

Not applicable.

\section{Competing interests}

The authors declare that they have no competing interests.

\section{References}

1. Coca-Pelaz A, Rodrigo JP, Bradley PJ, Vander Poorten V, Triantafyllou A, Hunt JL, Strojan P, Rinaldo A, Haigentz M Jr, Takes RP, et al: Adenoid cystic carcinoma of the head and neck - An update. Oral Oncol 51: 652-661, 2015.

2. International Head And Neck Scientific Group: Cervical lymph node metastasis in adenoid cystic carcinoma of the sinonasal tract, nasopharynx, lacrimal glands and external auditory canal: A collective international review. J Laryngol Otol 130: 1093-1097, 2016 
3. Togashi Y, Dobashi A, Sakata S, Sato Y, Baba S, Seto A, Mitani H, Kawabata K and Takeuchi K: MYB and MYBL1 in adenoid cystic carcinoma: Diversity in the mode of genomic rearrangement and transcripts. Mod Pathol 31: 934-946, 2018.

4. Shao GZ, Zhou RL, Zhang QY, Zhang Y, Liu JJ, Rui JA, Wei X and Ye DX: Molecular cloning and characterization of LAPTM4B, a novel gene upregulated in hepatocellular carcinoma. Oncogene 22: 5060-5069, 2003.

5. Liu X, Xiong F, Wei X, Yang H and Zhou R: LAPTM4B-35, a novel tetratransmembrane protein and its PPRP motif play critical roles in proliferation and metastatic potential of hepatocellular carcinoma cells. Cancer Sci 100: 2335-2340, 2009.

6. Liu XR, Zhou RL, Zhang QY, Zhang Y, Jin YY, Lin M, Rui JA and Ye DX: Structure analysis and expressions of a novel tetratransmembrane protein, lysosoma-associated protein transmembrane 4 beta associated with hepatocellular carcinoma. World J Gastroenterol 10: 1555-1559, 2004.

7. Yang H, Xiong F, Qi R, Liu Z, Lin M, Rui J, Su J and Zhou R: LAPTM4B-35 is a novel prognostic factor of hepatocellular carcinoma. J Surg Oncol 101: 363-369, 2010.

8. Yang H, Xiong FX, Lin M, Yang Y, Nie X and Zhou RL: LAPTM4B-35 overexpression is a risk factor for tumor recurrence and poor prognosis in hepatocellular carcinoma. J Cancer Res Clin Oncol 136: 275-281, 2010.

9. Zhou L, He XD, Chen J, Cui QC, Qu Q, Rui JA and Zhao YP: Overexpression of LAPTM4B-35 closely correlated with clinicopathological features and post-resectional survival of gallbladder carcinoma. Eur J Cancer 43: 809-815, 2007.

10. Kang Y, Yin M, Jiang W, Zhang H, Xia B, Xue Y and Huang Y: Overexpression of LAPTM4B-35 is associated with poor prognosis in colorectal carcinoma. Am J Surg 204: 677-683, 2012.

11. Yin M, Li C, Li X, Lou G, Miao B, Liu X, Meng F, Zhang H, Chen X, Sun M, et al: Over-expression of LAPTM4B is associated with poor prognosis and chemotherapy resistance in stages III and IV epithelial ovarian cancer. J Surg Oncol 104: 29-36, 2011.

12. Yang Y, Yang H, McNutt MA, Xiong F, Nie X, Li L and Zhou R: LAPTM4B overexpression is an independent prognostic marker in ovarian carcinoma. Oncol Rep 20: 1077-1083, 2008.

13. Tang H, Tian H, Yue W, Li L, Li S, Gao C, Si L, Qi L and Lu M Overexpression of LAPTM4B is correlated with tumor angiogenesis and poor prognosis in non-small cell lung cancer. Med Oncol 31: 974, 2014

14. Kong F, Gao F, Chen J, Sun Y, Zhang Y, Liu H, Li X, Yang P, Zheng R, Liu G, et al: Overexpressed LAPTM4B-35 is a risk factor for cancer recurrence and poor prognosis in non-small-cell lung cancer. Oncotarget 7: 56193-56199, 2016.

15. Zhang H, Wei Q, Liu R, Qi S, Liang P, Qi C, Wang A, Sheng B, $\mathrm{Li} \mathrm{L}$ and $\mathrm{Xu}$ Y: Overexpression of LAPTM4B-35: A novel marker of poor prognosis of prostate cancer. PLoS One 9 e91069, 2014.
16. Meng FL, Yin MZ, Song HT, Yang H, Lou G and Zhou RL: LAPTM4B-35 overexpression is an independent prognostic marker in endometrial carcinoma. Int J Gynecol Cancer 20: 745-750, 2010.

17. Cheng X, Zheng Z, Bu Z, Wu X, Zhang L, Xing X, Wang X, $\mathrm{Hu}$ Y, Du H, Li L, et al: LAPTM4B-35, a cancer-related gene, is associated with poor prognosis in TNM stages I-III gastric cancer patients. PLoS One 10: e0121559, 2015.

18. Liu L, Xu X, Jing L, Zhou G, Cao Z, Han Y and Zhou R: Lysosomal-associated protein transmembrane 4 Beta-35 overexpression is a novel independent prognostic marker for gastric carcinoma. PLoS One 10: e0118026, 2015.

19. Amit M, Binenbaum Y, Sharma K, Ramer N, Ramer I, Agbetoba A, Glick J, Yang X, Lei D, Bjørndal K, et al: Incidence of cervical lymph node metastasis and its association with outcomes in patients with adenoid cystic carcinoma. An international collaborative study. Head Neck 37: 1032-1037, 2015.

20. Amit M, Binenbaum Y, Trejo-Leider L, Sharma K, Ramer N, Ramer I, Agbetoba A, Miles B, Yang X, Lei D, et al: International collaborative validation of intraneural invasion as a prognostic marker in adenoid cystic carcinoma of the head and neck. Head Neck 37: 1038-1045, 2015.

21. Šteiner P, Andreasen S, Grossmann P, Hauer L, Vaněček T, Miesbauerová M, Santana T, Kiss K, Slouka D and Skálová A: Prognostic significance of $1 \mathrm{p} 36$ locus deletion in adenoid cystic carcinoma of the salivary glands. Virchows Arch 473: 471-480, 2018.

22. Ferrarotto R, Mitani Y, Diao L, Guijarro I, Wang J, ZweidlerMcKay P, Bell D, William WN Jr, Glisson BS, Wick MJ, et al: Activating NOTCH1 mutations define a distinct subgroup of patients with adenoid cystic carcinoma who have poor prognosis, propensity to bone and liver metastasis, and potential responsiveness to Notch1 inhibitors. J Clin Oncol 35: 352-360, 2017.

23. Li ZR: LAPTM4B: A novel diagnostic biomarker and therapeutic target for hepatocellular carcinoma. In: Hepatocellular Carcinoma. Lau WY (ed). InTech Press, Melbourne, FL, pp1-34, 2012.

24. Yang H, Xiong F, Wei X, Yang Y, McNutt MA and Zhou R: Overexpression of LAPTM4B-35 promotes growth and metastasis of hepatocellular carcinoma in vitro and in vivo. Cancer Lett 294: 236-244, 2010.

This work is licensed under a Creative Commons Attribution-NonCommercial-NoDerivatives 4.0 International (CC BY-NC-ND 4.0) License. 